Check for updates

Cite this: RSC Adv., 2019, 9, 38568

Received 3rd September 2019 Accepted 19th November 2019

DOI: $10.1039 / c 9 r a 07030 c$

rsc.li/rsc-advances

\section{Novel single excitation dual-emission carbon dots for colorimetric and ratiometric fluorescent dual mode detection of $\mathrm{Cu}^{2+}$ and $\mathrm{Al}^{3+}$ ionst}

\author{
Jinping Song, ${ }^{a}$ Qi Ma, (D) *a Yequn Liu, ${ }^{\text {*b }}$ Yong Guo, (D) a Feng Feng ${ }^{a}$ \\ and Shaomin Shuang (iD ${ }^{c}$
}

In this study, dual-emission carbon dots (D-CDs) are synthesized via a simple one-step solvothermal treatment of red tea. The obtained D-CDs are characterized by XPS, IR, TEM, XRD, fluorescence and UVvis spectroscopy techniques. It is found that $D-C D$ s present a strong red fluorescence emission peak at $671 \mathrm{~nm}$ and weak blue fluorescence emission peak at $478 \mathrm{~nm}$ under the excitation wavelength of $410 \mathrm{~nm}$. The unique dual-emission properties of D-CDs provide great opportunities in ratiometric fluorescence sensing applications. The results show that $\mathrm{Cu}^{2+}$ ions can quench the fluorescence of the red emission band of D-CDs effectively, resulting in the disappearance of red fluorescence ultimately. Upon the addition of $\mathrm{Al}^{3+}$ ions, the fluorescence of blue emission band at $478 \mathrm{~nm}$ grows apparently, and the fluorescence color transforms gradually from red to orange, then to yellow-green. Based on these findings, a novel ratiometric fluorescence and colorimetric dual mode nanosensor is developed for simultaneous detection of $\mathrm{Cu}^{2+}$ and $\mathrm{Al}^{3+}$ ions. Regarding $\mathrm{Cu}^{2+}$ ions, the fluorescent detection linear range is $0.1-50 \mu \mathrm{M}$ with detection limit of $0.1 \mu \mathrm{M}$, and the colorimetric detection limit is estimated as 25 $\mu \mathrm{M}$. With regard to $\mathrm{Al}^{3+}$ ions, the fluorescent detection linear range is $0-20 \mu \mathrm{M}$ and $25-100 \mu \mathrm{M}$ with detection limit of $0.5 \mu \mathrm{M}$, and the colorimetric detection limit is $20 \mu \mathrm{M}$. Furthermore, the fluorescence response mechanisms of $\mathrm{Cu}^{2+}$ and $\mathrm{Al}^{3+}$ ions were discussed detailed. To the best of our current knowledge, this will be the first research work on the simultaneous determination of $\mathrm{Cu}^{2+}$ and $\mathrm{Al}^{3+}$ using $D-C D s$ as fluorescent probes.

\section{Introduction}

Carbon dots (CDs), as a kind of extraordinary fluorescent nanomaterial, have attracted significant research interest because of their special properties including low toxicity, good water solubility and stability, and adjustable luminescence wavelength. In view of these unique properties, CDs have been widely used in optical sensing, bioimaging, drug delivery, photocatalysis and so on., ${ }^{1,2}$ So far, considerable efforts have been made aiming to reduce the cost, simplify the preparation process, increase fluorescence quantum yield, or realize long wavelength adjustable emission of $\mathrm{CDs}^{3-7}$ However, preparations of CDs with the desired properties still face a crucial challenge, most of the reported CDs exhibit single-emission

${ }^{a}$ College of Chemistry and Environmental Engineering, Institute of Applied Chemistry, Shanxi Datong University, Datong, Shanxi, 037009, China.E-mail: maqihx@163.com; Fax: +86-352-7158185; Tel: +86-352-7158185

${ }^{b}$ Analytical Instrumentation Center, State Key Laboratory of Coal Conversion, Institute of Coal Chemistry, Chinese Academy of Sciences, Taiyuan 030001, China. E-mail: liuyequn@sxicc.ac.cn

'Institute of Environmental Science, Shanxi University, Taiyuan, 030006, China

$\dagger$ Electronic supplementary information (ESI) available. See DOI: $10.1039 / \mathrm{c} 9 \mathrm{ra} 07030 \mathrm{c}$ properties which can be affected by concentration, environment and excitation wavelength, limiting their applications seriously. Compared with the traditional single-emitting CDs (SCDs), dual-emitting CDs (D-CDs) usually display two fluorescence emission bands at single excitation wavelength, as a result, exhibiting some potential application advantages. ${ }^{8,9}$ One significant advantage is that D-CDs can effectively improve the sensitivity and accuracy of the assay method by serving as ratiometric fluorescent sensing probe. ${ }^{10-13}$ Moreover, it even may achieve the simultaneous fluorescent determination of two different analytes without any other substances. ${ }^{9,14}$ Therefore, it is quite important to design D-CDs and further explore their potential applications.

Up to now, ratiometric fluorescent nanoprobes based on DCDs can be constructed by forming nanohybrid composites with other nanoparticles. For examples, Yan's research group used metal-organic framework (MOFs) as a carrier and then combined with red CDs and green $\mathrm{Tb}^{3+}$ to obtain dual-emitting hybrid system, which could successfully realize visual colorimetric detection for water contents and the relative humidity. ${ }^{15}$ Hereafter, they further achieved the ratiometric fluorescence sensing for copper ions by utilizing dual-emitting nanohybrid composites based on blue zirconium MOFs and red CDs. ${ }^{16}$ By 
encapsulating doped CDs in the structure of Eu-MOFs, Chi et al. prepared a red/blue dual-emitting ratiometric fluorescence nanoprobe to detect water content. ${ }^{17}$ Shangguan's research group coupled successfully CDs on the surface of dye-linked silica nanoparticles to develop a class of dual-emission nanosystem. ${ }^{18}$ Results showed that the addition of $\mathrm{Cu}^{2+}$ ion could bring about obvious ratiometric fluorescence response owing to the recognition interaction from the terminal ethylenediamine groups on CDs surface. In Xiang's research works, ${ }^{19,20}$ they found that this dual-emission nanosystem based on CDs linked silica nanoparticles could be used to build another ratiometric fluorescence sensor through the inhibition or catalyzing the oxidation process of $\mathrm{KBrO}_{3}$. In addition, with the help of the dispersion and crosslinking capabilities of silica layer, Liu et al. obtained dual-emission nanocomposite with apparent coreshell structure via covering CDs to the external surface of nitride phosphor. ${ }^{21}$ Very recently, researchers also developed some ratiometric fluorescence nanohybrid sensors by combining CDs and gold nanoclusters. ${ }^{22,23}$ In such ratiometric sensing strategy, it not only allows accurate ratiometric fluorescence sensing, but also permits visual detection by the naked eye.

Besides the above-mentioned strategy, functional groups modification and heteroatomic doping also are efficient ways to develop the D-CDs ratiometric fluorescent nanoprobes. Yan grafted coumarin on the surface of CDs to obtain dual-emission CDs which could be used in the ratiometric fluorescence analysis of copper ions. ${ }^{24}$ Lei's team obtained nitrogen and phosphorous co-doped CDs with blue and green fluorescence dualemission bands by microwave treating of aniline, ethylene and phosphoric acid. ${ }^{25}$ In a recent work, Tan prepared $\mathrm{Cu}-$ doped D-CDs by one-pot solvothermal method and realized successfully the ratiometric fluorescence analysis of iron ions, $\mathrm{pH}$ and vitamin A acetate. ${ }^{9}$ It was considered that the 426 emission band was originated from the energy gap of CD inherent defects, while the 488 emission band was attributed to another energy gap configuration ( $\mathrm{d}-\mathrm{d}$ orbital) from doping $\mathrm{Cu}(\mathrm{II})$. Although these D-CDs demonstrated excellent ratiometric fluorescence signals response in the detection process of analytes. However, complex preprocessing procedure, long preparation time and the use of poisonous reagents will greatly limit their potential application and development. Therefore, it is very necessary to develop an easy and environmentally friendly method for the preparation of D-CDs.

In this research work, we synthesized successfully the fluorescent CDs with dual-emission property by one-pot solvothermal treating of red tea in acetone solvent. The synthesis process is easy, rapid and environmentally friendly. The obtained D-CDs displayed a weak blue fluorescence emission peak at $478 \mathrm{~nm}$ and a strong red fluorescence emission peak at $671 \mathrm{~nm}$ under the excitation wavelength of $410 \mathrm{~nm}$. Such special dual-emission property of D-CDs not only provides more chance for them in ratiometric fluorescence sensing application, but also gives possibility to realize simultaneous determination of two different analytes. As expected, as-synthesized D-CDs can realize the simultaneous detection of two metal ions. To the best of our current knowledge, it will be the first research work on simultaneous detection of $\mathrm{Cu}^{2+}$ and $\mathrm{Al}^{3+}$ ions based on $\mathrm{D}$ CDs.

\section{Experimental section}

\subsection{Materials and reagents}

Red tea (Da Hong Pao), green tea (Tie Guan Yin) and dark tea (Anhua brick tea) were purchased from local supermarkets. Formamide, ethanol, acetone, dimethyl sulfoxide (DMSO), $\mathrm{N}, \mathrm{N}$ dimethylformamide (DMF), acetonitrile, tetrahydrofuran (THF), dichloromethane were obtained from Aladdin Reagents Company (Shanghai, China). All metal salts $\left(\mathrm{CuCl}_{2}, \mathrm{HgCl}_{2}\right.$, $\mathrm{NiCl}_{2}, \mathrm{MgCl}_{2}, \mathrm{ZnCl}_{2}, \mathrm{AlCl}_{3}, \mathrm{MnCl}_{2}, \mathrm{FeCl}_{2}, \mathrm{FeCl}_{3}, \mathrm{CrCl}_{3}, \mathrm{CaCl}_{2}$ and $\mathrm{CoCl}_{2}$ ) were acquired from Beijing Chemical Reagents Factory (Beijing, China). Ultrapure water was obtained by Millipore Milli-Q plus 185 water purification system and used in all experiments. All chemicals were analytical reagent grade and used directly.

\subsection{Characterization}

UV-vis absorption spectra were recorded on a PerkinElmer Lambda 35 UV-vis spectrometer. Fluorescence spectra were measured on a Cary Eclipse Fluorescence Spectrophotometer (Varian, USA). Fluorescence lifetime experiments were performed by an Edinburgh Instrument FLS-920 time-resolved/ steady state fluorescence spectrometer (Livingston, UK). X-ray photoelectron spectroscopy was measured using a PHI-5400 electron spectrometer. Transmission electron microscopy observations were carried out on a JEOL JEM-2100 transmission electron microscope. Fourier-transform infrared (FT-IR) spectra were recorded on a FTIR-650 spectrophotometer. Powder X-ray diffraction (XRD) analyses were performed on Bruker-D8 Focus diffractometer with $\mathrm{Cu} \mathrm{K} \alpha$ radiation. Dynamic light scattering (DLS) was measured by a Zeta plus particle size analyzer instrument (Brookhaven, USA).

\subsection{Preparation of D-CDs}

Typically, $300 \mathrm{mg}$ ground dry red tea powder was added into $10 \mathrm{~mL}$ acetone and stirred for $10 \mathrm{~min}$, and then the mixture solution was transferred into a $25 \mathrm{~mL}$ Teflon-lined stainless steel autoclave. The sealed autoclave was heated in oven at $180{ }^{\circ} \mathrm{C}$ for $1 \mathrm{~h}$. After cooling down to room temperature, the crude product was centrifuged at $10000 \mathrm{rpm}$ for $10 \mathrm{~min}$ to remove large particles. The crude product was further filtrated with $0.22 \mu \mathrm{m}$ filter membrane and then evaporated off the solvent at $50{ }^{\circ} \mathrm{C}$. The final product was redispersed in ethanol and stored at $4{ }^{\circ} \mathrm{C}$ for further characterization and use.

\subsection{Measurement of quantum yield}

The QY of D-CDs was detected by a relative method reported previously. The QY in the emission range of 600-800 $\mathrm{nm}$ was calculated using Rhodamine 6G (QY $=95 \%$ in ethanol) as the standard substance, and The QY in the emission range of 400$600 \mathrm{~nm}$ was calculated by employing quinine sulfate (in $0.1 \mathrm{M}$ $\mathrm{H}_{2} \mathrm{SO}_{4}$ ) as a standard. The relative QY values were estimated according to the following equation: 


$$
\Phi_{\mathrm{x}}=\Phi_{\text {std }} \frac{I_{\mathrm{x}}}{A_{\mathrm{x}}} \frac{A_{\text {std }}}{I_{\text {std }}} \frac{\eta_{\mathrm{x}}^{2}}{\eta_{\text {std }}^{2}}
$$

where the subscript std and $\mathrm{x}$ refer to the reference substance and the CDs, respectively. The $\Phi$ is the fluorescence QY. $I, A$ and $\eta$ are the integrated fluorescence intensity, the optical densities and the refractive index of the solvent, respectively. The optical density of all samples should be controlled to less than 0.10 at the excitation wavelength.

\section{5 $\mathrm{Cu}^{2+}$ and $\mathrm{Al}^{3+}$ ions sensing}

Detection of $\mathrm{Cu}^{2+}$ and $\mathrm{Al}^{3+}$ ions were preformed in ethanol solvent at room temperature on a Cary Eclipse Fluorescence Spectrophotometer. Typically, for the detection of $\mathrm{Cu}^{2+}, \mathrm{CuCl}_{2}$ ethanol solution with different concentration was added into $2 \mathrm{~mL}$ ethanol solution containing D-CDs $\left(0.2 \mathrm{mg} \mathrm{mL}^{-1}\right)$ and incubated for $60 \mathrm{~min}$. Then, the corresponding fluorescence emission spectrum was recorded under $410 \mathrm{~nm}$ excitation wavelength. For the detection of $\mathrm{Al}^{3+}$, the corresponding fluorescence spectra were measured after reacting for $1 \mathrm{~min}$. The fluorescence quenching ability of $\mathrm{Cu}^{2+}$ and $\mathrm{Al}^{3+}$ ions in other solvents was measured by the similar experiment procedure except that the solvent was replaced.

\subsection{Selectivity experiment}

To explore the selectivity of the constructed sensor, the fluorescent responses of twelve kinds of metal ions $\left(\mathrm{Cr}^{3+}, \mathrm{Al}^{3+}, \mathrm{Cu}^{2+}\right.$, $\mathrm{Fe}^{3+}, \mathrm{Fe}^{2+}, \mathrm{Zn}^{2+}, \mathrm{Co}^{2+}, \mathrm{Mg}^{2+}, \mathrm{Ni}^{2+}, \mathrm{Ca}^{2+}, \mathrm{Mn}^{2+}$ and $\mathrm{Hg}^{2+}$ ) were examined under the same experimental conditions as the mentioned above. The concentrations of all metal ions were 100 $\mu \mathrm{M}$.

\section{Results and discussion}

\subsection{Synthesis and characterization of D-CDs}

D-CDs is prepared facilely by one-step solvothermal treatment of red tea in acetone. In order to obtain the desirable products, massive experiments have been carried out to optimize the experimental parameters, and the corresponding results were displayed in Tables S1 and S2 in ESI. $\dagger$ It is worth noting that solvent plays a key role in forming D-CDs. In water and formamide media, single-emission blue CDs can be obtained, but in ethanol and acetone solvents, D-CDs are easier to be formed (see Fig. S1 in ESI $\dagger$ ). Furthermore, the type of tea would have a certain influence on the QYs of CDs. By comparison, we find that D-CDs obtained by solvothermal treatment of red tea in acetone give the relative high QY. Under the optimal reaction temperature $\left(180{ }^{\circ} \mathrm{C}\right)$ and reaction time $(1 \mathrm{~h})$, the QY of red emission band of the D-CDs is the highest (6.06\%). In addition, as depicted in Table $\mathrm{S} 3, \uparrow$ the as-synthesized D-CDs exhibit different QY in different solvents. The QY of red emission band can reach $8.93 \%$ in DMF.

Fig. 1 displays the TEM images of D-CDs at different magnifications and the corresponding particles size distribution. It is observed that the particle sizes of D-CDs are mostly distributed in the range of 1.9-4.1 $\mathrm{nm}$ and the average
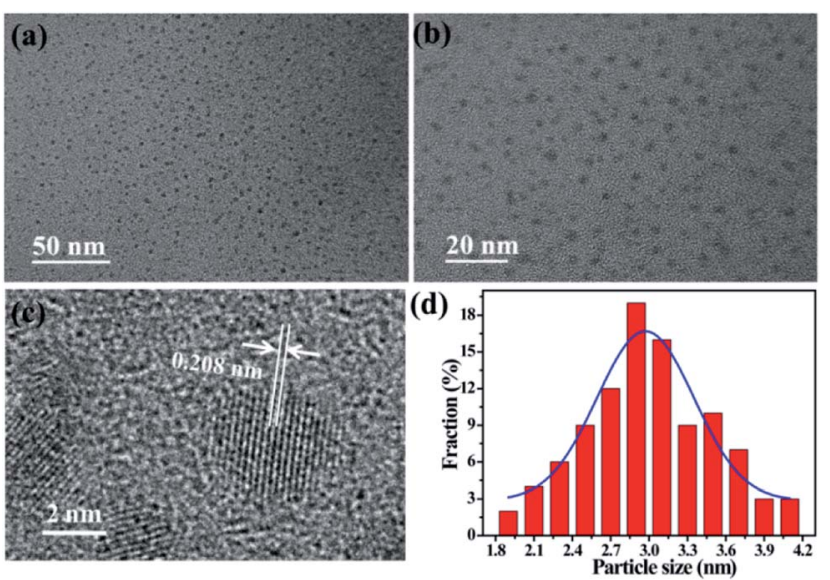

Fig. 1 TEM images of D-CDs (a) scale bar is $50 \mathrm{~nm}$ and (b) scale bar is $20 \mathrm{~nm}$. (c) HRTEM image of D-CDs (scale bar is $2 \mathrm{~nm}$ ). (d) Size distribution of D-CDs.

diameter is $2.9 \mathrm{~nm}$ (ca. 19\%). The size distribution conforms to the Gaussian feature, and the half width is $0.9 \mathrm{~nm}$, suggesting that the obtained $\mathrm{D}$-CDs possess a narrow size distribution. HRTEM image in Fig. 1c shows that D-CDs exhibit quasi-spherical shape and have well dispersion. It clearly displays the particle possess well-resolved lattice fringe, a clear lattice spacing of $0.208 \mathrm{~nm}$ may originate from the (110) lattice plane of graphite. ${ }^{26}$

In order to determine the elemental composition and chemical structures of the D-CDs, XPS and FT-IR are carried out. As depicted in the full scan spectrum (Fig. 2a), the three characteristic peaks located at 532, 400 and $284 \mathrm{eV}$ are ascribed to O1s, N1s and C1s, respectively. This implies that the as-synthesized D-CDs mainly compose of carbon, nitrogen, oxygen elements. The C1s spectrum of D-CDs can be deconvoluted into five peaks at 288.5, 286.4, 285.3, 284.5 and $283.3 \mathrm{eV}$ (Fig. 2b), representing that $\mathrm{D}-\mathrm{CDs}$ contain $\mathrm{C}=\mathrm{O}$, $\mathrm{C}-\mathrm{OH}, \mathrm{C}-\mathrm{N}, \mathrm{C}-\mathrm{C} / \mathrm{C}=\mathrm{C}$ and $\mathrm{C}-\mathrm{H}$ bonds, respectively. ${ }^{27-29}$ In high-resolution spectrum of O1s (Fig. 2c), the two fitting peaks at 532.7 and $532.0 \mathrm{eV}$ are assigned to $\mathrm{C}-\mathrm{OH} / \mathrm{C}-\mathrm{O}-\mathrm{C}$ and $\mathrm{C}=\mathrm{O}$ groups. ${ }^{30} \mathrm{~N} 1 \mathrm{~s}$ can be resolved into four peaks at 401.4, $400.5,399.7$ and $398.0 \mathrm{eV}$ that are attributed to graphitictype $\mathrm{N}, \mathrm{N}-\mathrm{H}$, pyrrolic-type $\mathrm{N}$ and pyridinic-type $\mathrm{N}$ groups (Fig. 2d). ${ }^{28,30}$ These XPS attributions are further verified by FT-IR analysis (Fig. 2e). The broad bands at 3431 and $3190 \mathrm{~cm}^{-1}$ originate from the $\mathrm{O}-\mathrm{H}$ and $\mathrm{N}-\mathrm{H}$ stretching vibrations. The two small bands situated at 2857 and $2921 \mathrm{~cm}^{-1}$ are assigned to the $\mathrm{C}-\mathrm{H}$ stretching vibrations. ${ }^{28}$ The vibration bands at 1701 and $1647 \mathrm{~cm}^{-1}$ suggest the presence of $\mathrm{C}=\mathrm{O}$ bonds. Furthermore, peaks at 1567 and $1401 \mathrm{~cm}^{-1}$ are attributed to the $\mathrm{C}=\mathrm{C}$ and $\mathrm{C}-\mathrm{N}$ stretching vibration, and the peaks around 1136 and $1096 \mathrm{~cm}^{-1}$ are ascribed to the $\mathrm{C}-\mathrm{O}$ stretching vibration and the bending vibrations of $\mathrm{C}-\mathrm{H} \cdot{ }^{30,31}$ In addition, the XRD pattern (Fig. 2f) of D-CDs displays a wide diffraction peak centered at $21.2^{\circ}$, which suggests the formation of highly disordered carbon phase. $^{32}$ 

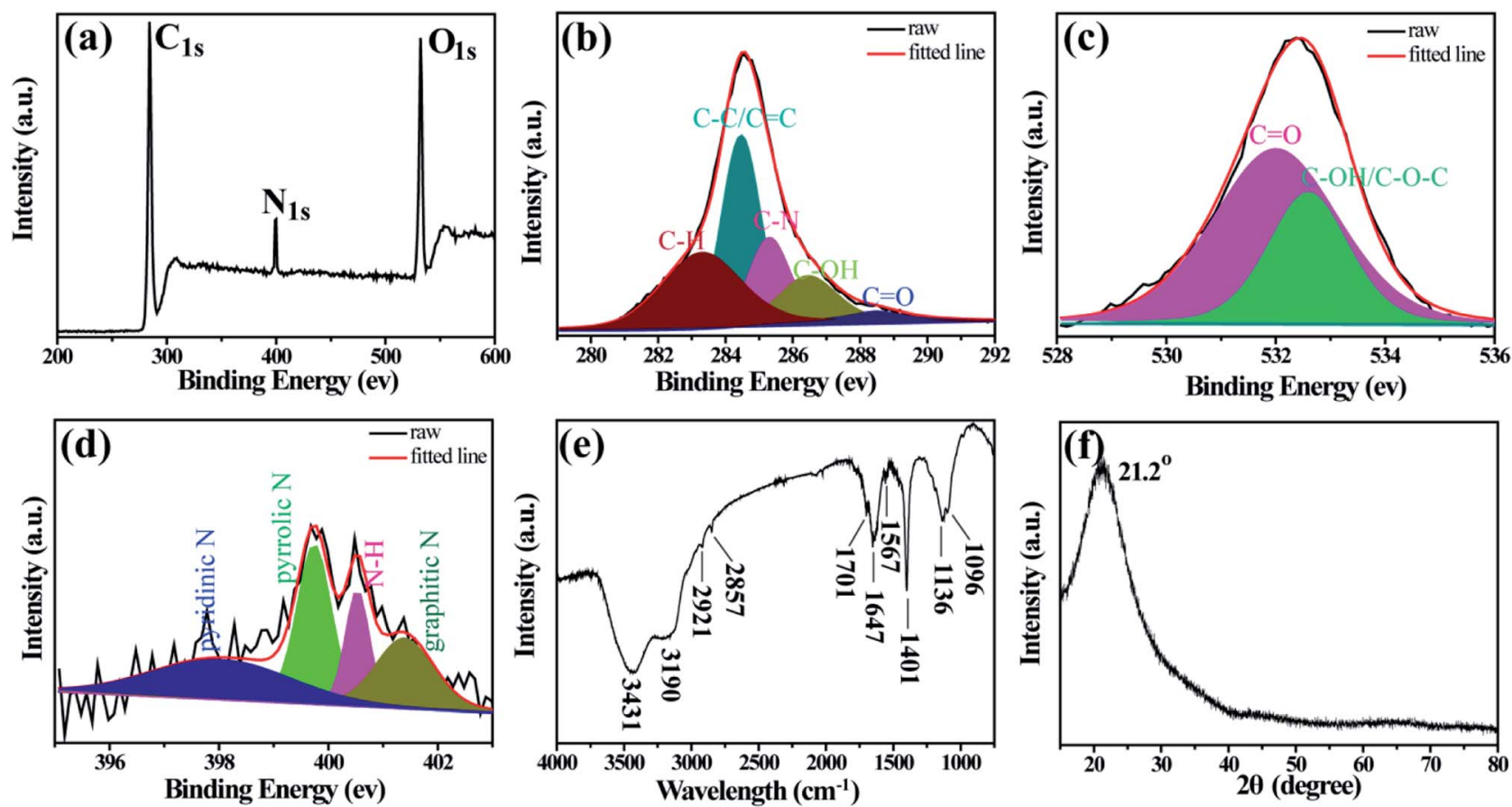

Fig. 2 (a) XPS survey spectra of D-CDs. High-resolution C1s (b), O1s (c), and N1s (d) spectra of D-CDs. (e) FTIR spectra of the D-C Ds. (f) XRD of D-CDs.

\subsection{Optical properties of D-CDs}

The optical properties of the as-prepared D-CDs are studied by UV-vis absorption and fluorescence techniques. From Fig. 3, it can be seen that the UV-vis absorption spectrum of D-CDs exhibits four absorption bands at 273, 411, 503 and $665 \mathrm{~nm}$. The absorption band at $273 \mathrm{~nm}$ is considered to be the typical $\pi$ $\rightarrow \pi^{*}$ transition characteristic peak of the $\mathrm{C}=\mathrm{C}$ group. The bands at 411, 503 and $665 \mathrm{~nm}$ are possibly originated from $\pi \rightarrow$ $\pi^{*}$ and $\mathrm{n} \rightarrow \pi^{*}$ transitions of the $\mathrm{C}=\mathrm{O}$ or $\mathrm{C}=\mathrm{N}$ groups. ${ }^{28,33}$ More surprisingly, under $410 \mathrm{~nm}$ excitation wavelength, D-CDs

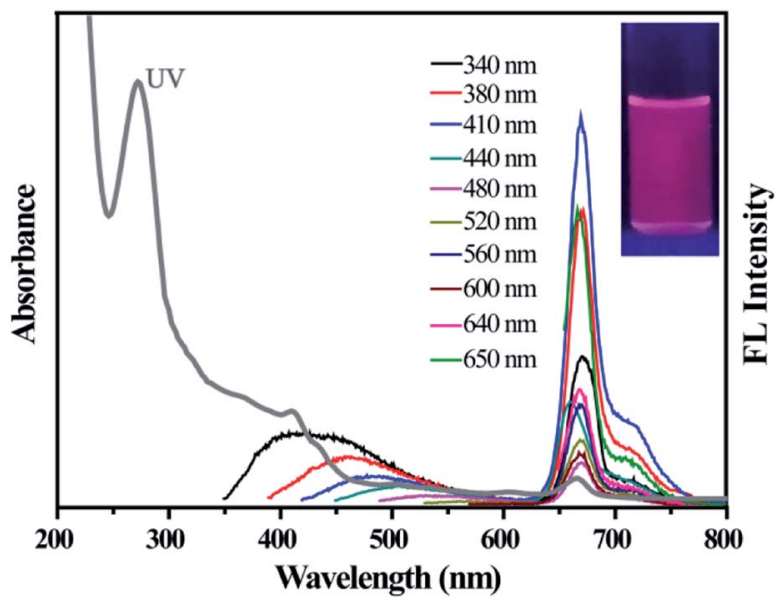

Fig. 3 UV-visible absorption (gray curve) and FL emission spectra of $D$-CDs (excitation wavelengths range from 340 to $650 \mathrm{~nm}$ ). The inset is photographs of D-CDs under UV lamp. demonstrate two separated FL emission peaks at $478 \mathrm{~nm}$ and $671 \mathrm{~nm}$, which correspond to the blue-emission and redemission bands, respectively. From the fluorescence spectra, it is further found that the blue band exhibits an apparent excitation-dependent feature when the excitation wavelength is increased from 340 to $480 \mathrm{~nm}$. Previous research pointed that it is possible originate from the intrinsic and defects emission, which is considered to be related to oxygenous-related defect states. But the red emission band presents excitationindependent emission properties, which probably originates from the nitrogen-containing fluorophores (e.g. porphyrin molecules) ${ }^{34,35}$ Moreover, it is also observed that the fluorescence intensity of the blue-emission band is much weaker than those of the red-emission band. It is well known that the naked eye is much more sensitive to red light than to the blue one. Thus, the resultant D-CDs ethanol suspension seems to emit bright red fluorescence under $365 \mathrm{~nm}$ UV lamp (see the inset in Fig. 3). As depicted in Fig. S2, $\dagger$ as the excitation wavelength varies from 340 to $600 \mathrm{~nm}$, the CIE color coordinates can be shifted from blue region $(0.2137,0.1845)$ to the red region $(0.7181,0.2719)$. This indicates that the as-synthesized D-CDs maybe have potential application as an optical conversion material.

\section{3 $\mathrm{Cu}^{2+}$-induced red-emission quenching and $\mathrm{Al}^{3+}$-induced blue-emission enhancement of D-CDs}

It is well known that fluorescent CDs have been widely applied as a versatile fluorescent nanoprobe to detect various metal ions based on the metal ions induced FL quenching mechanism. ${ }^{36,37}$ So, we are very curious to know if the as-prepared D-CDs will 
exhibit the similar response. To examine metal ion responding ability towards D-CDs, the corresponding fluorescence spectra with and without twelve kinds of metal ions $\left(100 \mu \mathrm{M} \mathrm{Cr}^{3+}, \mathrm{Al}^{3+}\right.$, $\mathrm{Cu}^{2+}, \mathrm{Fe}^{3+}, \mathrm{Fe}^{2+}, \mathrm{Zn}^{2+}, \mathrm{Co}^{2+}, \mathrm{Mg}^{2+}, \mathrm{Ni}^{2+}, \mathrm{Ca}^{2+}, \mathrm{Mn}^{2+}$ and $\left.\mathrm{Hg}^{2+}\right)$ are measured, respectively. Surprisingly, we observe that the presence of $\mathrm{Cu}^{2+}$ ion can cause significant $\mathrm{FL}$ quenching of the redemission band of D-CDs, on the contrary, $\mathrm{Al}^{3+}$ leads to the manifest FL enhancement and slight red-shift of the blueemission band of D-CDs (Fig. S3†), whereas there are no obvious changes for other ten kinds of metal ions. The specific coordinates in the CIE chromaticity diagram move from the red light area $(0.42,0.29)$ to white light area $(0.34,0.28)$ and bluegreen area $(0.19,0.31)$ upon addition of $\mathrm{Cu}^{2+}$ and $\mathrm{Al}^{3+}$ ions, respectively (Fig. $\mathrm{S} 4 \dagger$ ). Under $410 \mathrm{~nm}$ excitation wavelength, 100 $\mu \mathrm{M}$ of $\mathrm{Cu}^{2+}$ ion can result in approximately 14 times of FL quenching ability at $671 \mathrm{~nm}$, and $100 \mu \mathrm{M}$ of $\mathrm{Al}^{3+}$ ion can bring about nearly 24 times of FL enhancement at $478 \mathrm{~nm}$ (Fig. 4a). Such significant FL changes in the blue color fluorescence enhancement and red color fluorescence quench suggest the high selectivity of the dual-emission D-CDs towards $\mathrm{Al}^{3+}$ and $\mathrm{Cu}^{2+}$ ions, which allows to fabricate a simple analytical method for the simultaneous determination of $\mathrm{Al}^{3+}$ and $\mathrm{Cu}^{2+}$ ions.

In order to detect $\mathrm{Al}^{3+}$ and $\mathrm{Cu}^{2+}$ ions effectively, the experimental conditions including the solvents and reaction time are optimized. Fig. 4b displays that the FL intensity of D-CDs at $478 \mathrm{~nm}$ is basically constant in the presence of $\mathrm{Al}^{3+}$ ions in DMSO, DMF, acetonitrile, THF and water, however, the FL intensity of D-CDs is significantly increased $\sim 24$-fold and $\sim 12$ fold in ethanol and acetone solvents, respectively. Similarly, in the ethanol media, the presence of $\mathrm{Cu}^{2+}$ ions can cause the maximum FL quenching at $671 \mathrm{~nm}$ (Fig. 4c). Therefore, the ethanol solvent is selected as the optimal reaction media in the following experiments. Next, the effect of the reaction time is further investigated and shown in Fig. S5 and S6. $\dagger$ Result exhibits that FL signal response of the D-CDs is dependent of the reaction time. Upon addition of $\mathrm{Al}^{3+}$ ion, the FL intensity of D-CDs at $478 \mathrm{~nm}$ increases to the maximum after 2 min incubation, then decrease slightly with time. With regards to $\mathrm{Cu}^{2+}$ ion, the FL intensity of D-CDs at $671 \mathrm{~nm}$ decreases rapidly within the initial $20 \mathrm{~min}$, then this decrease becomes slowly and reaches the constant at $60 \mathrm{~min}$. Considering the speed and easyoperation of the detection assay, the optimal reaction time is selected as $2 \mathrm{~min}$ and $60 \mathrm{~min}$ for $\mathrm{Al}^{3+}$ and $\mathrm{Cu}^{2+}$ detection, respectively.

The blue emission peak of D-CDs at $478 \mathrm{~nm}$ grows gradually upon increasing the concentration of $\mathrm{Al}^{3+}$ ions, while the intensity change of red emission peak at $671 \mathrm{~nm}$ is negligible. $\ln \left(F_{478} / F_{671}\right)$ exhibits good linearity with the concentration of $\mathrm{Al}^{3+}$ in range of 0 to $20 \mu \mathrm{M}$ and 25 to $100 \mu \mathrm{M}$, respectively (Fig. 5a). The correlation equation is $\ln \left(F_{478} / F_{671}\right)=0.1273 C$ $(\mu \mathrm{M})-3.1261(R=0.9972)$, and $\ln \left(F_{478} / F_{671}\right)=0.007070 C(\mu \mathrm{M})$ - $0.4070(R=0.9860)$, respectively, in which $F_{478}$ and $F_{671}$ signify the FL intensity of D-CDs at $478 \mathrm{~nm}$ and $671 \mathrm{~nm}$ in the presence of $\mathrm{Al}^{3+}$ separately. The detection limit is $0.5 \mu \mathrm{M}$ at 3 times signal-to-noise ratio. It is noted that the FL color of D-CD solution varies from red to orange, then to yellow-green after adding different concentration of $\mathrm{Al}^{3+}$ ions and can be observed by naked eye under UV lamp (365 nm) (Fig. 5b). The FL color of D-CDs solution becomes obvious different after the addition of $20 \mu \mathrm{M}$, implying that the colorimetric detection limit for $\mathrm{Al}^{3+}$ is $20 \mu \mathrm{M}$.

The fluorescence intensity of the red emission peak gradually declines upon increasing the concentration of $\mathrm{Cu}^{2+}$ ions. The relative fluorescence intensity $\left(F / F_{0}\right)$ displays good linearity with the concentration of $\mathrm{Cu}^{2+}$ ion (Fig. 6a). The linearity equation is $F / F_{0}=-0.01814 C(\mu \mathrm{M})+0.9873(R=0.9958)$, the linearity range is from 0.1 to $50 \mu \mathrm{M}$ and the detection limit is 0.1 $\mu \mathrm{M}$. As depicted in Fig. 6b, the red FL quenching can be seen obviously by naked eye in the presence of $25 \mu \mathrm{M} \mathrm{Cu}^{2+}$, indicating that the colorimetric detection limit for $\mathrm{Cu}^{2+}$ is $25 \mu \mathrm{M}$.

\subsection{Response mechanism of D-CDs to $\mathrm{Al}^{3+}$ and $\mathrm{Cu}^{2+}$}

Previous reports demonstrate fluorescence response of CDs to metal ions mainly involve the fluorescence resonance energy transfer (FRET), inner filter effect (IFE), aggregation-induced emission quenching (ACQ) and aggregation-induced emission enhancement (AIEE).$^{38-41}$ In our case, it is impossible that there are FRET and IFE between D-CDs and $\mathrm{Al}^{3+}$ or $\mathrm{Cu}^{2+}$ since two
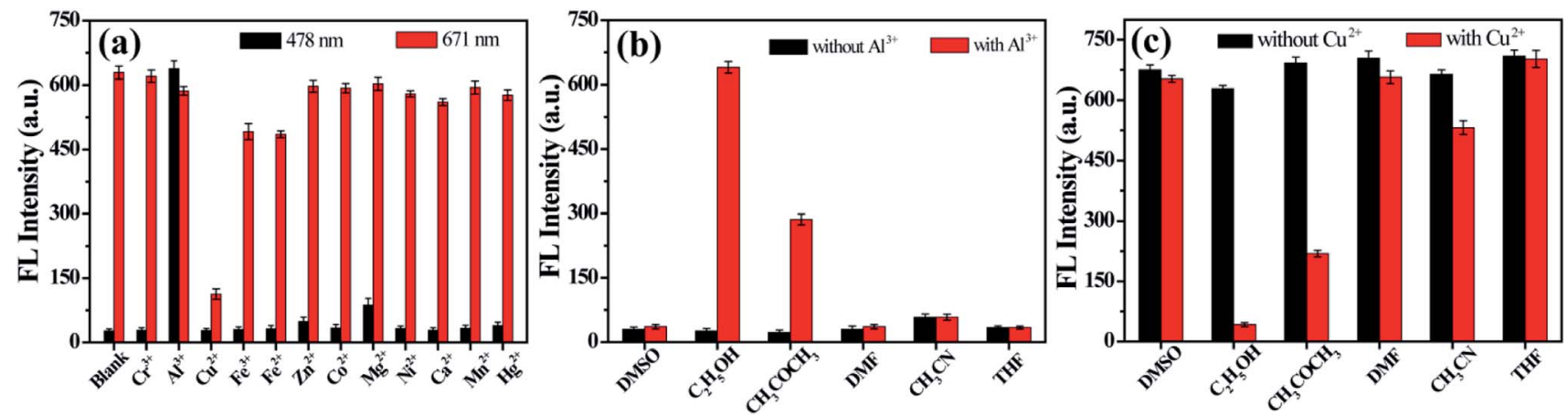

Fig. 4 (a) The FL intensity histogram of D-CDs in ethanol in the absence and presence of different metal ions (the excitation wavelength $\lambda_{\text {ex }}$ is $410 \mathrm{~nm}$. Black and red column signify $478 \mathrm{~nm}$ and $671 \mathrm{~nm}$ emission intensity). (b) The FL intensity of D-CDs at $478 \mathrm{~nm}$ in different solvents (black column and red column represent without and with $\mathrm{Al}^{3+}$ ions, respectively). (c) The FL intensity of D-CDs at $671 \mathrm{~nm}$ in different solvents (black column and red column represent without and with $\mathrm{Cu}^{2+}$ ions, respectively). 

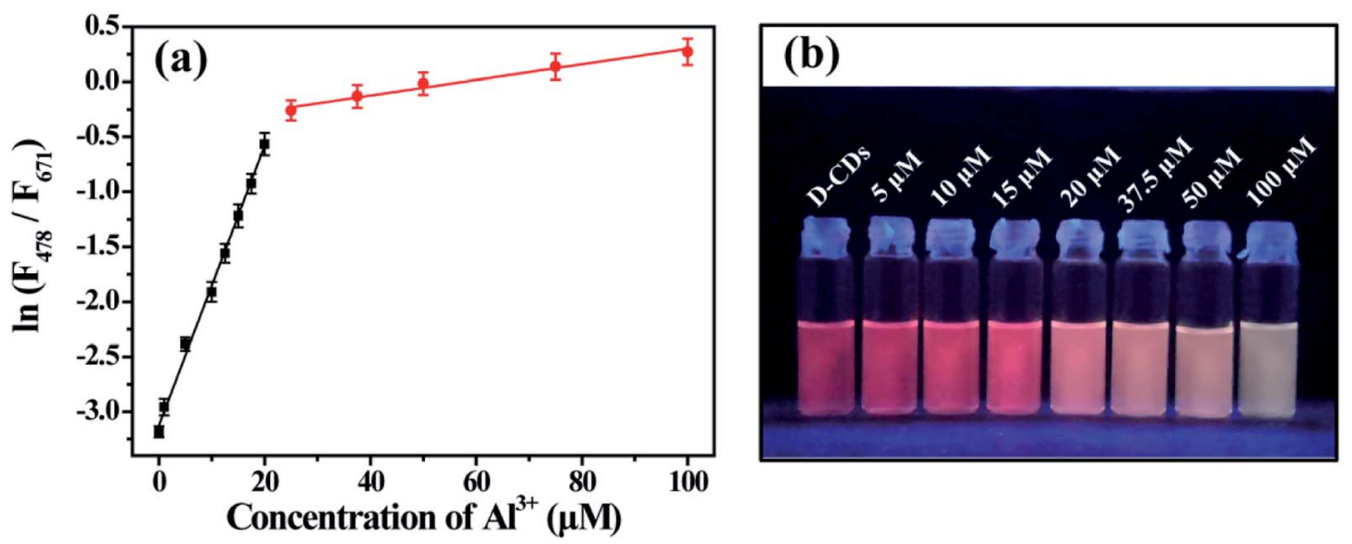

Fig. 5 (a) The linear plot between $\ln \left(F_{478} / F_{671}\right)$ and the concentration of $\mathrm{Al}^{3+}$ in the range of $0-2.0 \times 10^{-5} \mathrm{M}$ and $2.5 \times 10^{-5} \mathrm{M}$ to $1.0 \times 10^{-4} \mathrm{M}$. (b) Visual FL photographs of D-CDs in the presence of different concentrations of $\mathrm{Al}^{3+}$ under $365 \mathrm{~nm}$ UV light.

kinds of metal ions have neither characteristic absorption nor spectral overlap with the excitation and emission spectra of DCDs (Fig. S7 $\dagger$ ). Generally, ACQ and AIEE are related to the aggregation of CDs caused by temperature, solvent and metal ions. ${ }^{42}$ Thus, to investigate whether the FL response mechanism of D-CDs after binding with $\mathrm{Al}^{3+}$ or $\mathrm{Cu}^{2+}$ are ascribed to the aggregation-induced emission effects, we measured the DLS of D-CDs with and without different metal ions. As displayed the DLS data in Fig. S8, $\uparrow$ the diameter of the D-CDs in ethanol distributes in the range of $2.1-15.5 \mathrm{~nm}$. It is observed that the diameters of D-CDs in the presence of other ten kinds of metal ions are basically close. In contrast, when $\mathrm{Al}^{3+}$ or $\mathrm{Cu}^{2+}$ ions are added, the diameter of the D-CDs are increased clearly, and the size distributions are in range of $25-300 \mathrm{~nm}$ (the average particle size is $68.7 \mathrm{~nm}$ ) and $15-165 \mathrm{~nm}$ (the average particle size is $49.8 \mathrm{~nm}$ ), respectively, verifying the formation of larger DCDs aggregates. For $\mathrm{Cu}^{2+}$ ions, the aggregation of D-CDs can be attributed to the coordination of $\mathrm{Cu}^{2+}$ ions with the surface $-\mathrm{NH}_{2}$ sites, which will make nanoparticles closer, as a result, obvious ACQ effect occurs. The detailed quenching process is further discussed by the time-resolved fluorescence technique.
When the excitation and emission wavelengths is 410 and $670 \mathrm{~nm}$, the average lifetime of D-CDs is $7.98 \mathrm{~ns}$ and can be fitted into two components: $6.57 \mathrm{~ns}(c a .92 .7 \%)$ and $25.84 \mathrm{~ns}$ ( $c a$. $7.3 \%$ ). After mixing with $\mathrm{Cu}^{2+}$ ions, the $\mathrm{D}-\mathrm{CDs} / \mathrm{Cu}^{2+}$ lifetime is 7.91 ns (Fig. S9†). Further analysis demonstrates that the quenching mechanism of $\mathrm{Cu}^{2+}$ ions towards D-CDs follows a static process. The Stern-Volmer constant $\left(K_{\mathrm{sv}}\right)$ is $2.56 \times 10^{4} \mathrm{~L}$ $\mathrm{mol}^{-1}$ and the biomolecular reaction rate constant $\left(K_{\mathrm{q}}\right)$ is 3.18 $\times 10^{12} \mathrm{~L} \mathrm{~mol}^{-1} \mathrm{~s}^{-1}$.

For $\mathrm{Al}^{3+}$ ions, based on the obvious size increase, the FL enhancement of the blue-band of D-CDs is considered as the result of the AIEE effects. The FL decay behavior and the corresponding quantum yield of the D-CDs/Al ${ }^{3+}$ system are displayed in Fig. S10 and Table S4. $\dagger$ Upon the addition of $\mathrm{Al}^{3+}$ ions, the lifetime of blue-band of D-CDs increases from $3.83 \mathrm{~ns}$ to $4.80 \mathrm{~ns}$, and the quantum yield also increases from $0.80 \%$ to $8.01 \%$ (quinine sulfate as a standard). The radiative rate constant $\left(k_{\mathrm{r}}\right)$ and nonradiative rate constant $\left(k_{\mathrm{nr}}\right)$ are calculated through the following formula: $k_{\mathrm{r}}=\Phi / \tau$ and $k_{\mathrm{nr}}=(1-\Phi) / \tau$, respectively. Apparently, owing to the change in lifetime and quantum yields, the $k_{\mathrm{r}}$ value increase from $2.09 \times 10^{6} \mathrm{~s}^{-1}$ to
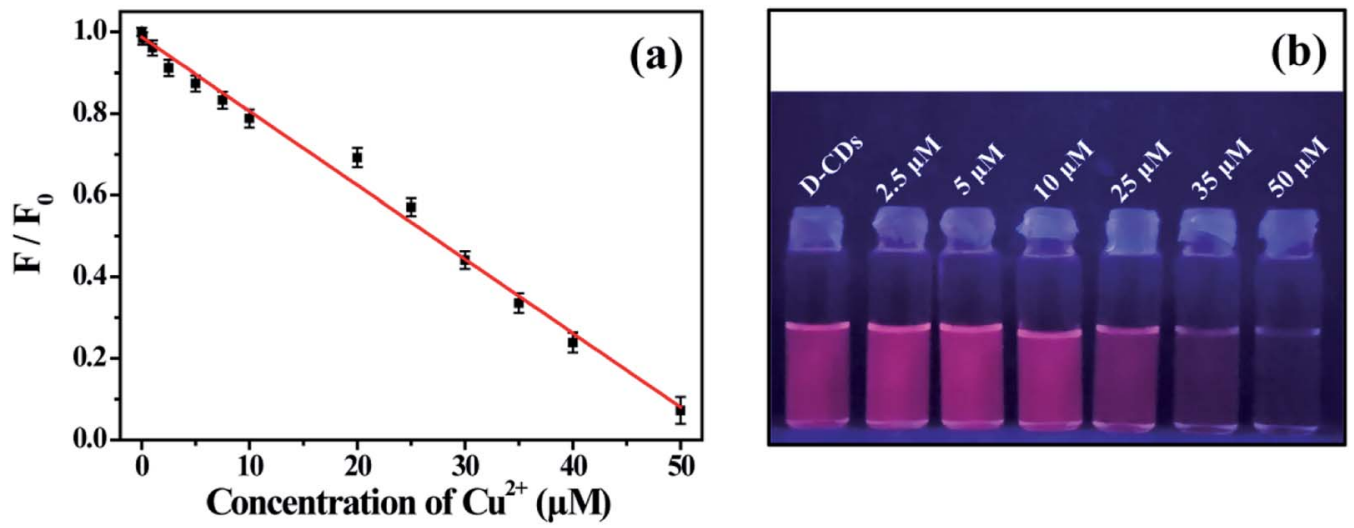

Fig. 6 (a) The linear plot between $F / F_{0}$ and the concentration of $\mathrm{Cu}^{2+}$ in the range of $0-5.0 \times 10^{-5} \mathrm{M}\left(F\right.$ and $F_{0}$ signify the FL intensity of $D$ - $C D$ s at $671 \mathrm{~nm}$ in the presence and absence of $\mathrm{Cu}^{2+}$ ). (b) Visual FL photographs of D-CDs in the presence of different concentrations of $\mathrm{Cu}^{2+}$ under $365 \mathrm{~nm}$ UV light. 
$16.69 \times 10^{6} \mathrm{~s}^{-1}$, while the $k_{\mathrm{nr}}$ value decreases from $2.59 \times 10^{8}$ $\mathrm{s}^{-1}$ to $1.92 \times 10^{8} \mathrm{~s}^{-1}$. Moreover, the enhanced emission of blueband of D-CDs in glycerol solution with high viscous further reveals the rotational hindering of the surface groups on D-CDs (Fig. S11 $\dagger$ ) blocks the non-radiative decay, leading to improved FL intensity. ${ }^{\mathbf{4 3 , 4 4}}$

\section{Conclusions}

In summary, CDs obtained from one-step solvothermal treatment of red tea exhibits special dual-emission property under single-wavelength excitation. This endows CDs multiple sensing ability, which can be employed to realize the simultaneous detection of $\mathrm{Cu}^{2+}$ and $\mathrm{Al}^{3+}$ without any interference effect. Based on the obvious FL color and FL intensity variations, a novel colorimetric and ratiometric fluorescence dual mode nanosensor for simultaneous detection of $\mathrm{Cu}^{2+}$ and $\mathrm{Al}^{3+}$ ions is developed. Further studies demonstrate that the introduction of $\mathrm{Cu}^{2+}$ and $\mathrm{Al}^{3+}$ ions can cause the aggregates of D-CDs. It is confirmed that red band $(671 \mathrm{~nm})$ fluorescence quenching of $\mathrm{D}$ CDs in the presence of $\mathrm{Cu}^{2+}$ ions originated from ACQ effect. However, the obvious fluorescence enhancement of the blue band ( $c a .470 \mathrm{~nm}$ ) of D-CDs is ascribed to AIEE effect owing to the rotational hindering of D-CDs, reducing the non-radiative decay. This research will open our mind to exploit multiemission CDs with more charming multiple sensing properties.

\section{Conflicts of interest}

There are no conflicts to declare.

\section{Acknowledgements}

This work was supported by the National Natural Science Foundation of China (21301111 and 51602322), Research Project Supported by Shanxi Scholarship Council of China (2016-105) and the Research Foundation of Datong City (2019027 and 2017129).

\section{Notes and references}

1 S. Y. Lim, W. Shen and Z. Q. Gao, Chem. Soc. Rev., 2015, 44, 362-381.

2 G. A. M. Hutton, B. C. M. Martindale and E. Reisner, Chem. Soc. Rev., 2017, 46, 6111-6123.

3 S. Zhu, Q. Meng, L. Wang, J. Zhang, Y. Song, H. Jin, K. Zhang, H. Sun, H. Wang and B. Yang, Angew. Chem., Int. Ed., 2013, 125, 4045-4049.

4 Q. Xu, T. Kuang, Y. Liu, L. Cai, X. Peng, T. S. Sreeprasad, P. Zhao, Z. Yu and N. Li, J. Mater. Chem. B, 2016, 4, 72047219.

5 H. Ding, S. B. Yu, J. S. Wei and H. M. Xiong, ACS Nano, 2016, 10, 484-491.

6 J. Chen, J. S. Wei, P. Zhang, X. Q. Niu, W. Zhao, Z. Y. Zhu, H. Ding and H. M. Xiong, ACS Appl. Mater. Interfaces, 2017, 9, 18429-18433.
7 J. Ge, Q. Jia, W. Liu, L. Guo, Q. Liu, M. Lan, H. Zhang, X. Meng and P. Wang, Adv. Mater., 2015, 27, 4169-4177.

8 J. Zhao, M. Huang, L. Zhang, M. Zou, D. Chen, Y. Huang and S. Zhao, Anal. Chem., 2017, 89, 8044-8049.

9 P. P. Zhu, Z. Cheng, L. L. Du, Q. Chen and K. J. Tan, Langmuir, 2018, 34, 9982-9989.

10 J. Liu, Y. Dong, Y. Ma, Y. Han, S. Ma, H. Chen and X. Chen, Nanoscale, 2018, 10, 13589-13598.

11 R. Wang, X. Wang and Y. Sun, Microchim. Acta, 2017, 184, 187-193.

12 L. Lu, C. Feng, J. Xu, F. Wang, H. Yu, Z. Xu and W. Zhang, Biosens. Bioelectron., 2017, 92, 101-108.

13 M. Lan, J. Zhang, Y. S. Chui, P. Wang, X. Chen, C. S. Lee, H. L. Kwong and W. Zhang, ACS Appl. Mater. Interfaces, 2014, 6, 21270-21278.

14 L. Yu, G. Ren, M. Tang, B. Zhu, F. Chai, G. Li and D. Xu, Eur. J. Inorg. Chem., 2018, 3418-3426.

15 J. X. Wu and B. Yan, Dalton Trans., 2017, 46, 7098-7105.

16 J. X. Wu and B. Yan, Dalton Trans., 2017, 46, 15080-15086.

17 Y. Q. Dong, J. H. Cai, Q. Q. Fang, X. You and Y. W. Chi, Anal. Chem., 2016, 88, 1748-1752.

18 X. J. Liu, N. Zhang, T. Bing and D. H. Shangguan, Anal. Chem., 2014, 86, 2289-2296.

19 G. Q. Xiang, Y. Ren, H. Zhang, H. H. Fan, X. M. Jiang, L. J. He and W. J. Zhao, Can. J. Chem., 2018, 96, 72-77.

20 G. Q. Xiang, Y. Ren, Y. Xia, W. J. Mao, C. Fan, S. Y. Guo, P. P. Wang, D. H. Yang, L. J. He and X. M. Jiang, Spectrochim. Acta, Part A, 2017, 177, 153-157.

21 Y. H. Chen, B. F. Lei, M. T. Zheng, H. R. Zhang, J. L. Zhuang and Y. L. Liu, Nanoscale, 2015, 7, 20142-20148.

22 H. Z. Xie, J. Dong, J. L. Duan, G. I. N. Waterhouse, J. Y. Hou and S. Y. Ai, Sens. Actuators, B, 2018, 259, 1082-1089.

23 Y. S. He, C. G. Pan, H. X. Cao, M. Z. Yue, L. Wang and G. X. Liang, Sens. Actuators, B, 2018, 265, 371-377.

24 F. Yan, Z. Bai, Y. Chen, F. Zu, X. Li, J. Xu and L. Chen, Sens. Actuators, B, 2018, 275, 86-94.

25 X. C. Sun, C. Brücknerb and Y. Lei, Nanoscale, 2015, 7, 17278-17282.

26 Y. Q. Dong, R. X. Wang, H. Li, J. W. Shao, Y. W. Chi, X. M. Lin and G. N. Chen, Carbon, 2012, 50(8), 2810-2815.

27 X. J. Gong, W. J. Lu, M. C. Paau, Q. Hu, X. Wu, S. M. Shuang, C. Dong and M. F. F. Choi, Anal. Chim. Acta, 2015, 861, 74-84.

28 L. Pan, S. Sun, L. Zhang, K. Jiang and H. W. Lin, Nanoscale, 2016, 8(39), 17350-17356.

29 X. W. Qie, M. H. Zan, P. Miao, L. Li, Z. M. Chang, M. F. Ge, P. Gui, Y. G. Tang and W. F. Dong, J. Mater. Chem. B, 2018, 6(31), 3549-3554.

30 L. Luo, P. Wang, Y. H. Wang and F. Wang, Sens. Actuators, B, 2018, 273, 1640-1677.

31 Y. F. Chen, Y. Y. Wu, B. Weng, B. Wang and C. M. Li, Sens. Actuators, B, 2016, 223, 689-696.

32 Y. L. He, J. L. He, Z. H. Yu, H. R. Zhang, Y. L. Liu, G. Q. Hu, M. T. Zheng, H. W. Dong, J. L. Zhuang and B. F. Lei, J. Mater. Chem. C, 2018, 6, 2495-2501.

33 W. Wang, Y. M. Li, L. Cheng, Z. Q. Cao and W. G. Liu, J. Mater. Chem. B, 2014, 2(1), 46-48. 
34 T. X. Zhang, J. Y. Zhu, Y. Zhai, H. Wang, X. Bai, B. Dong, H. Y. Wang and H. W. Song, Nanoscale, 2017, 9(35), 1304213051.

35 L. P. Li, R. P. Zhang, C. X. Lu, J. H. Sun, L. J. Wang, B. T. Qu, T. T. Li, Y. D. Liu and S. J. Li, J. Mater. Chem. B, 2017, 5(35), 7328-7334.

36 N. Wang, Y. T. Wang, T. T. Guo, T. Yang, M. L. Chen and J. H. Wang, Biosens. Bioelectron., 2016, 85, 68-75.

37 B. F. Shi, Y. B. Su, L. L. Zhang, M. J. Huang, R. J. Liu and S. L. Zhao, ACS Appl. Mater. Interfaces, 2016, 8(17), 1071710725.

38 W. L. Gao, H. H. Song, X. Wang, X. Q. Liu, X. B. Pang, Y. M. Zhou, B. Gao and X. J. Peng, ACS Appl. Mater. Interfaces, 2018, 10(1), 1147-1154.
39 Y. B. Wang, Q. Chang and S. L. Hu, Sens. Actuators, B, 2017, 253, 928-933.

40 H. X. Zhao, L. Q. Liu, Z. D. Liu, Y. Wang, X. J. Zhao and C. Z. Huang, Chem. Commun., 2011, 47(9), 2604-2606.

41 B. B. Chen, R. S. Li, M. L. Liu, H. Z. Zhang and C. Z. Huang, Chem. Commun., 2017, 53(36), 4958-4961.

42 C. Li, W. J. Liu, X. B. Sun, W. Pan, G. F. Yu and J. P. Wang, Sens. Actuators, B, 2018, 263, 1-9.

43 Y. Hong, J. W. Y. Lam and B. Z. Tang, Chem. Soc. Rev., 2011, 40(11), 5361-5388.

44 M. X. Gao, C. F. Liu, Z. L. Wu, Q. L. Zeng, X. X. Yang, W. B. Wu, Y. F. Li and C. Z. Huang, Chem. Commun., 2011, 49(73), 8015-8017. 